\title{
Optimization of Production Planning Using Goal Programming Approach at Chocolate Factory
}

\author{
Noviyarsi. ${ }^{1,}$, Lestari Setiawati $^{1}$, and Arhamna Arisya ${ }^{1}$ \\ ${ }^{1}$ Industrial Engineering Department, Bung Hatta University, Padang, Indonesia
}

\begin{abstract}
Company $\mathrm{X}$ is a mini chocolate factory in West Sumatera Province. The Company produces three types of product, that is paralin chocolate, bar chocolate $75 \mathrm{gr}$ and bar chocolate 20 gr. Today, production planning only based on customer demand without any analysis an optimized amount of production to maximize profit. The purposed of this research was the optimization of production planning to maximize company profit. Goal programming approach was used in this research. There were four goals that need to optimize, that are, production volume, profit, processing time and raw material. Horizon plan was set up for six periods. LINDO software was used to compute an optimized result of goal programming. The final result shows an average of production amount for six periods, that were paralin chocolate about $65,95 \mathrm{~kg}$, bar chocolate 75 gr about 883 bars and bar chocolate 20 gr about 3725 bars with maximum profit about Rp. 17.806.782,-
\end{abstract}

\section{Introduction}

Production planning is related to the planning of the type and quantity of products to be produced by the company. Production planning aims to achieve production and labor stability against demand fluctuations. Company $\mathrm{X}$ is a small industry engaged in the processing of chocolate. There are 3 products produced are paralin chocolate, 75 gram chocolate bar and 20 gram chocolate bar. At this time, the determination of production quantities and types of products to be produced only based on demand. The problem faced by the company was cannot fulfill customer demand. The company had never done production planning to predict the availability of raw materials, labor and time to meet consumer demand. It does not affect the optimal level of production that can maximize profits. This is due to the availability of cocoa stock which is still dependent on the cocoa harvest season, the imbalance in the capacity of the cocoa processing machine and the productivity of the employee working time.

Goal programming is one mathematical model that can be used in optimizing production planning with multiple goals. Goal Programming is one of the mathematical models that is viewed in accordance with multi-purpose problem solving because through its deviation variable, goal programming automatically captures information about the relative achievement of the objectives [1]. The only difference lies in the presence of a pair of deviation variables that appear on the purpose function and constraint functions [2]. This

* Corresponding author: lestarisetiawati@bunghatta.ac.id 
model requires various inputs (inputs) from existing production systems in the factory to support the decision to be generated. Therefore goal programming is suitable to solve production planning problems at the chocolate factory. Based on this, the purpose of this research was to optimize the production to produce maximum profit.

\section{Reseacrh Methodology}

\subsection{Data Collection}

The data collected in the form of primary data and secondary data. Primary data is obtained by doing self-observation while secondary data obtained from company document. The data required in this studied were production data, production cost data, processing time data and demand data.

\subsection{Aggregation}

Aggregation had been done to facilitate forecasting production. This is because the products produced by the factory used the same raw materials, production processes, machinery, and production equipment. The resulting chocolate product is distinguished only on the basis of the mold and the weight of the product. The aggregate calculation of chocolate products had been done by combining the product based on the unit of measure or weight of each product.

\subsection{Forecasting}

Forecasting was used to predict amount of demand in the future. Forecasting had been done using Win QSB software for six periods.

\subsection{Disaggregation}

The disaggregation calculation is performed to determine the production forecasting result of each chocolate product. The disaggregation calculation is done by percentage approach by multiplying the forecast result by the proportion of each product.

\subsection{Goal Programming}

In preparing a goal programming model must first determine the decision variables of the optimization model, then formulate the function constraints and objective functions to be achieved. In the goal programming model there is a deviation variable in the constraint function. These variables serve to accommodate the deviation of the completion of the objectives to be achieved. In the process of processing the model, the number of devisional variables will be minimized in the objective function [3].

\subsubsection{Decision Variables}

$X_{i t} \quad$ : Amount of production product- $i$ at period- $t$

$i \quad$ : type of product. $i=1,2,3$

$t \quad$ : production period. $i=1,2,3,4,5,6$ 

a. Production volume
b. Profit
c. Processing time capacity
d. Raw material availability

\subsubsection{Goal Programming Formulation}

$\operatorname{Min} \mathrm{Z}=\left(\mathrm{d}_{1}^{+}+\mathrm{d}_{1}^{-}+\mathrm{d}_{2}^{+}+\mathrm{d}_{2}^{-}+\ldots .+\mathrm{dn}^{+}+\mathrm{d}_{\mathrm{n}}{ }^{-}\right)$

\section{Result and Conclusion}

\subsection{Aggregation}

Table 1 shows result of aggregation from three types of chocolate product.

Table 1. Product Aggregation

\begin{tabular}{|c|c|c|c|c|c|c|c|c|}
\hline \multirow[b]{2}{*}{ Période } & \multicolumn{3}{|c|}{ Produc } & \multicolumn{3}{|c|}{ Agrégat } & \multirow[b]{2}{*}{$\begin{array}{c}\text { Sum } \\
\text { Agrégat } \\
\text { (gram) }\end{array}$} & \multirow[b]{2}{*}{$\begin{array}{c}\text { Sum } \\
\text { Agrégat } \\
\text { (kg) }\end{array}$} \\
\hline & $\begin{array}{c}\text { Pralin } \\
\text { (kg) }\end{array}$ & $\begin{array}{l}\text { Bar } \\
75 \text { gr } \\
\text { (unit) }\end{array}$ & $\begin{array}{l}\text { bar } \\
20 \text { gr } \\
\text { (unit) }\end{array}$ & $\begin{array}{l}\text { Collât } \\
\text { Pralin } \\
\text { (gram) }\end{array}$ & $\begin{array}{c}\text { Collât } \\
\text { Batang } \\
75 \text { gr } \\
\text { (gram) }\end{array}$ & $\begin{array}{c}\text { Collât } \\
\text { Batang } \\
20 \text { gr } \\
\text { (gram) }\end{array}$ & & \\
\hline 1 & 81 & 582 & 2182 & 81000 & 43650 & 43640 & 168290 & 169 \\
\hline 2 & 91 & 657 & 2466 & 91000 & 49275 & 49320 & 189595 & 190 \\
\hline 3 & 91 & 560 & 2101 & 91000 & 42000 & 42020 & 175020 & 176 \\
\hline 4 & 84 & 607 & 2277 & 84000 & 45525 & 45540 & 175020 & 176 \\
\hline 5 & 104 & 752 & 2819 & 104000 & 56400 & 56380 & 216780 & 217 \\
\hline 6 & 110 & 789 & 2961 & 110000 & 59175 & 59220 & 228395 & 229 \\
\hline Total & 562 & 3948 & 14804 & 561000 & 296025 & 296120 & 1153245 & 1157 \\
\hline
\end{tabular}

\subsection{Forecasting Result}

Table 2 shows forecasting result for 6 period for each product. Forecasting had been done using Win $Q S B$ software.

Table 2. Forecasting Result for Product

\begin{tabular}{|c|c|c|c|}
\hline Periods & Praline & Bar 75 gram & Bar 20 gram \\
\hline 1 & 111,15 & 799 & 2995 \\
\hline 2 & 116,06 & 838 & 3146 \\
\hline 3 & 131,39 & 809 & 3034 \\
\hline 4 & 126,48 & 914 & 3429 \\
\hline 5 & 131,68 & 953 & 3570 \\
\hline 6 & 137,44 & 986 & 3700 \\
\hline
\end{tabular}

\subsection{Goal Programming Formulation}

$\operatorname{Min} \mathrm{Z}=\left(\mathrm{d}_{1}{ }^{+}+\mathrm{d}_{1}{ }^{-}+\mathrm{d}_{2}{ }^{+}+\mathrm{d}_{2}{ }^{-}+\mathrm{d}_{3}{ }^{+}+\mathrm{d}_{3}{ }^{-}+\mathrm{d}_{4}{ }^{+}+\mathrm{d}_{4}{ }^{-}+\mathrm{d}_{5}{ }^{+}+\mathrm{d}_{5}{ }^{-}+\mathrm{d}_{6}{ }^{+}+\mathrm{d}_{6}{ }^{-}+\mathrm{d}_{7}{ }^{+}+\mathrm{d}_{7}{ }^{-}+\mathrm{d}_{8}{ }^{+}+\right.$ $\mathrm{d}_{8}{ }^{-}+\mathrm{d}_{9}{ }^{+}+\mathrm{d}_{9}{ }^{-}+\mathrm{d}_{10}{ }^{+}+\mathrm{d}_{10^{-}}+\mathrm{d}_{11}{ }^{+}+\mathrm{d}_{11^{-}}+\mathrm{d}_{12}{ }^{+}+\mathrm{d}_{12}{ }^{-}+\mathrm{d}_{13}{ }^{+}+\mathrm{d}_{13^{-}}+\mathrm{d}_{14}{ }^{+}+\mathrm{d}_{14^{-}}+\mathrm{d}_{15}{ }^{+}+\mathrm{d}_{15^{-}}+$ 


$$
\begin{aligned}
& \left.\mathrm{d}_{16}{ }^{+}+\mathrm{d}_{16^{-}}+\mathrm{d}_{17}{ }^{+}+\mathrm{d}_{17^{-}}+\mathrm{d}_{18}{ }^{+}+\mathrm{d}_{18^{-}}\right)+\left(\mathrm{d}_{19^{-}}+\mathrm{d}_{20^{-}}+\mathrm{d}_{21^{-}}+\mathrm{d}_{22^{-}}+\mathrm{d}_{23^{-}}+\mathrm{d}_{24^{-}}\right)+\left(\mathrm{d}_{25}{ }^{+}+\mathrm{d}_{25^{-}}+\right. \\
& \mathrm{d}_{26}{ }^{+}+\mathrm{d}_{26}{ }^{-}+\mathrm{d}_{27}{ }^{+}+\mathrm{d}_{27}{ }^{-}+\mathrm{d}_{28}{ }^{+}+\mathrm{d}_{28}{ }^{-}+\mathrm{d}_{29}{ }^{+}+\mathrm{d}_{29}{ }^{-}+\mathrm{d}_{30}{ }^{+}+\mathrm{d}_{30}{ }^{-}+\mathrm{d}_{31}{ }^{+}+\mathrm{d}_{31}{ }^{-}+\mathrm{d}_{32}{ }^{+}+\mathrm{d}_{32}{ }^{-}+\mathrm{d}_{33^{+}} \\
& \left.+\mathrm{d}_{33^{-}}+\mathrm{d}_{34}{ }^{+}+\mathrm{d}_{34^{-}}+\mathrm{d}_{35}{ }^{+}+\mathrm{d}_{35^{-}}+\mathrm{d}_{36}{ }^{+}+\mathrm{d}_{36}{ }^{-}\right)+\left(\mathrm{d}_{37}{ }^{+}+\mathrm{d}_{37^{-}}+\mathrm{d}_{38}{ }^{+}+\mathrm{d}_{38}{ }^{-}+\mathrm{d}_{39}{ }^{+}+\mathrm{d}_{39^{-}}+\mathrm{d}_{40}{ }^{+}+\right. \\
& \mathrm{d}_{40}{ }^{-}+\mathrm{d}_{41}{ }^{+}+\mathrm{d}_{41^{-}}+\mathrm{d}_{42}{ }^{+}+\mathrm{d}_{42}{ }^{-}+\mathrm{d}_{43}{ }^{+}+\mathrm{d}_{43^{-}}+\mathrm{d}_{44^{+}}+\mathrm{d}_{44^{-}}+\mathrm{d}_{45}{ }^{+}+\mathrm{d}_{45}{ }^{-}+\mathrm{d}_{46}{ }^{+}+\mathrm{d}_{46}{ }^{-}+\mathrm{d}_{47}{ }^{+}+\mathrm{d}_{47^{-}} \\
& +\mathrm{d}_{48}{ }^{+}+\mathrm{d}_{48}{ }^{-}+\mathrm{d}_{49}{ }^{+}+\mathrm{d}_{49}{ }^{-}+\mathrm{d}_{50}{ }^{+}+\mathrm{d}_{50}{ }^{-}+\mathrm{d}_{51}{ }^{+}+\mathrm{d}_{51}{ }^{-}+\mathrm{d}_{52}{ }^{+}+\mathrm{d}_{52}{ }^{-}+\mathrm{d}_{53}{ }^{+}+\mathrm{d}_{53}{ }^{-}+\mathrm{d}_{54}{ }^{+}+\mathrm{d}_{54}{ }^{-}+ \\
& \mathrm{d}_{55}{ }^{+}+\mathrm{d}_{55}{ }^{-}+\mathrm{d}_{56}{ }^{+}+\mathrm{d}_{56}{ }^{-}+\mathrm{d}_{57}{ }^{+}+\mathrm{d}_{57^{-}}+\mathrm{d}_{58}{ }^{+}+\mathrm{d}_{58}{ }^{-}+\mathrm{d}_{59}{ }^{+}+\mathrm{d}_{59}{ }^{-}+\mathrm{d}_{60}{ }^{+}+\mathrm{d}_{60}{ }^{-}+\mathrm{d}_{61}{ }^{+}+\mathrm{d}_{61}{ }^{-}+\mathrm{d}_{62}{ }^{+} \\
& \left.+\mathrm{d}_{62}{ }^{-}+\mathrm{d}_{63}{ }^{+}+\mathrm{d}_{63}{ }^{-}+\mathrm{d}_{64}{ }^{+}+\mathrm{d}_{64}{ }^{-}+\mathrm{d}_{65}{ }^{+}+\mathrm{d}_{65}{ }^{-}+\mathrm{d}_{66}{ }^{+}+\mathrm{d}_{66}{ }^{-}\right)
\end{aligned}
$$

Constraint :

1. Demand

$$
\sum_{i=1}^{t} \sum_{j=1}^{n}\left(d_{1}^{-}-d_{1}^{+}=D_{i j}\right)
$$

2. Profit

$$
\sum_{i=1}^{t}\left(X_{i}+d_{1}^{-}-d_{1}^{+}=P_{i}\right)
$$

3. Processing time

$$
\sum_{i=1}^{t}\left(X_{i}+d_{1}^{-}-d_{1}^{+}=T_{i}\right)
$$

4. Cocoa

$$
\sum_{i=1}^{t} \sum_{j=1}^{n}\left(X_{i j}+d_{1}^{-}-d_{1}^{+}=C_{j}\right)
$$

5. Sugar

$$
\sum_{i=1}^{t} \sum_{j=1}^{n}\left(X_{i j}+d_{1}^{-}-d_{1}^{+}=S_{j}\right)
$$

6. Milk

$$
\sum_{i=1}^{t} \sum_{j=1}^{n}\left(X_{i j}+d_{1}^{-}-d_{1}^{+}=M_{j}\right)
$$

7. Lestin

$$
\sum_{i=1}^{t} \sum_{j=1}^{n}\left(X_{i j}+d_{1}^{-}-d_{1}^{+}=L_{j}\right)
$$

Where :
$i \quad$ : time period
j : number of product
$X \quad$ : Production Plan
$d_{1}^{-} \quad$ : deviation variable if performance below production target
$d_{1}^{-} \quad$ : deviaton variable if performance above production target
$D_{i j} \quad:$ Demand product $j$ at period $i$
$P_{i} \quad$ : Total profit at period $i$
$T_{i} \quad$ : Total processing time at period $i$ 


$$
\begin{array}{ll}
C_{i} & : \text { Total Cocoa at period } i \\
S_{i} & : \text { Total Sugar at period } i \\
M_{i} & : \text { Total Milk at period } i \\
L_{i} & : \text { Total Lestin at period } i
\end{array}
$$

The solution of the goal programming model shows that for the optimum fulfillment of chocolate demand of chocolate in periods 4,5 and 6 . In brown stems $75 \mathrm{gr}$ per optimum period and chocolate stems $20 \mathrm{gr}$ per period is not optimal, the average optimization of paralin chocolate production is $65,93 \mathrm{~kg}, 75 \mathrm{~kg}$ stem chocolate as much as 883 stems and $20 \mathrm{gr}$ bar chocolate as much as 3725 stems. The optimal advantage that can be achieved from the goal programming model solution is an average of Rp. 17,806,782 per period. For the utilization rate of time consuming process will be optimal with average optimization of 230.3 hours per period. For the consumption level of brown fat, brown paste, sugar, milk, lecithin and soda as a whole will be below the level of availability and above the availability level or there is excess stock and shortage of raw material stock. From the average goal programming model optimization solution for the use of brown fat raw material as much as $55,80 \mathrm{~kg}$, chocolate pasta $51,66 \mathrm{~kg}$, sugar 51,66 kg, milk 45,47 kg, lecithin $0,91 \mathrm{~kg}$ and soda as much as $0.14 \mathrm{~kg}$.

\section{References}

1. Charles, Optimization Modelling With Lingo, Lindo System, Inc, Chicago, (2006)

2. Siswanto, A, Goal Programming dengan Menggunakan LINDO, Gramedia, Jakarta, (2007)

3. Anis, M. Nandiroh, S. dan Utami, A.D, Optimasi Perencanaan Produksi Dengan Metode Goal Programming, Jurnal Ilmiah Teknik Industri. Vol.5, No.3: page 133-143 (2007) 